\title{
Phenotype of acid sphingomyelinase deficiency knockout mice
}

\section{Dear Editor,}

In their letter, 'Niemann-Pick Disease versus Acid Sphingomyelinase Deficiency', Lozano et al. ${ }^{1}$ summarize reports favoring the role of acid sphingomyelinase and ceramides as lipid signaling molecules in pathways leading to apoptosis. The authors also address in the letter our studies described in Cell Death and Differentiation. ${ }^{2}$

Lozano et al. ${ }^{1}$ report that mouse embryonic fibroblasts (MEFs) derived from acid sphingomyelinase (aSMase)deficient mice display a normal sphingomyelin content, normal morphology and doubling times, and a morphology and distribution of caveolae comparable to wild-type cells. ${ }^{1}$ Analyses of 8-week-old asmase ${ }^{-1-}$ did not show hepatic or spleenic accumulation of sphingomyelin, lymphopenia, or alterations of proliferatory and apoptotic responses of $T$ cells in comparison to wild-type mice, which in the author's view, differ from our results recently published in this journal. $^{2}$

The crucial point in the comparison of the two studies is that the experiments described by Lozano et al. are not comparable to ours, although studying several of the parameters described in our publication, for the following reasons. A major characteristic of both the human Niemann-Pick disease (NPD) ${ }^{3}$ and, likewise, aSMase deficiency in mice generated by gene targeting, is the age-dependent accumulation of sphingomyelin in lysosomes and subcellular membranes of asmase ${ }^{-1-}$ cells. ${ }^{3-5}$ The onset of the clinical manifestations of NPD, and, likewise, in aSMase-deficient mice, such as tremors, apathy, or leukopenia (which refers here to a loss of phagocytotically active leukocytes rather than lymphocytes (M Nix and W Stoffel, unpublished results)) is 12-16 weeks of age and later. The experiments described in our publication have been performed with mice from our asmase $^{-1-}$ mouse line ${ }^{4}$ of at least 12-16 weeks of age and older which have developed the histological and clinical manifestations of NPD: the alterations of the lipid composition of hepatocyte, splenocyte and fibroblast cell membranes as well as the consequences for cellular signal transduction pathways which parallel the increasing incorporation of sphingomyelin and the changes of the complex lipid composition of the bilayer of plasmamembranes. ${ }^{2}$

Young asmase ${ }^{-1-}$ mice are phenotypically indistinguishable from wild-type mice ${ }^{4,5}$ and MEFs derived from asmase $^{-1-}$ mice initially divide normally. With increasing number of passages, MEFs severely accumulate sphingomyelin, show a prolonged cell cycle, and eventually die after the sixth or seventh passage ${ }^{1,2}$ (M Nix and W Stoffel, unpublished observations). To analyze alterations of the membrane lipid composition or the morphology of rafts and caveolae in MEFs of aSMase deficient mice, it is therefore essential to examine cells which have developed the NPD phenotype. Lorenzo et al., ${ }^{1}$ however, used 8-week-old asmase $^{-1-}$ mice and embryonic fibroblasts (MEGs) from asmase $^{-1-}$ mice.

We considered it only appropriate to analyze the biochemical consequences of this genetic asmase ${ }^{-1-}$ model beyond postnatal weeks 12-16, in which their phenotypical characteristics have developed as a consequence of the lipid storage and the changes of the lipid bilayer of the plasmamembrane with the associated functional perturbations described in our publication. We regard this difference (rather than the minor one in the substrains of the 129 line between the asmase ${ }^{-1-}$ mouse line first generated in our laboratory ${ }^{4}$ and the one used by Lorezo et al..$^{5}$ ) as the reason for the discrepancy.

W Stoffel ${ }^{\star, 1}$

${ }^{1}$ Laboratory of Molecular Neuroscience, Institute of Biochemistry, Faculty of Medicine, University of Cologne, D-50931 Cologne, Germany ${ }^{\star}$ Corresponding author: E-mail: Wilhelm.Stoffel@uni-koeln.de

1. Lozano J et al. (2000) Cell Death Differ., this issue

2. Nix M and Stoffel W (2000) Cell Death Differ. 7: 413-424

3. Brady R (1987) Sphingomyeline lipidosis: Niemann-Pick disease. In Metabolic Basis of Inherited Disease, Stanbury JB, Wyngarden, Fredrickson DS, Goldstein $\mathrm{JL}$ and Brown MS, eds (New York: McGraw-Hill) pp. 831-841

4. Otterbach B and Stoffel W (1995) Cell 81: 1053-1061

5. Horinouchi K et al. (1995) Nat. Genet. 10: 288-293 